\title{
Atenuación pragmática: el caso de las condicionales $^{1}$
}

PRAGMATIC MITIGATION: THE CASE OF CONDITIONALS

\section{Marianna Chodorowska-Pilch}

University of Southern California

Resumen

Este trabajo estudia las estructuras condicionales cuya finalidad es atenuar la fuerza del contenido proposicional de determinados actos de habla. Su uso se debe a la percepción del hablante, quien sigue las normas de cortesía, establecidas por Lakoff (1973) y Brown y Levinson (1978, 1987), con el objetivo de mitigar la imposición de sus enunciados. El análisis de las grabaciones de una agencia de viajes en Valencia (España) indica que el significado atenuante de las condicionales o cuasicondicionales se puede inferir solo en el contexto de las afirmaciones impositivas, explicaciones, preguntas, peticiones indirectas, sugerencias y ofertas.

PALABRAS CLAVE: estructuras condicionales, cortesía, mitigación

\section{Abstract}

This study analyzes conditional structures whose purpose is to mitigate the force of the propositional content in specific speech acts. Their use is based on the speaker's perception, who following politeness norms, established by Lakoff (1973), and Brown and Levinson (1978, 1987), intends to attenuate the imposition of their utterances. The analysis of recordings done in a travel agency in Valencia (Spain) indicates that the attenuating meaning of the conditionals or semi-conditionals can be inferred only in

\footnotetext{
${ }^{1}$ Muchas gracias a las editoras por la paciencia y a Marta Albelda por su ayuda en la parte técnica y por su apoyo durante la última etapa. Todos los defectos y omisiones son la responsabilidad de la misma autora. Este trabajo fue presentado en el Congreso Internacional de Atenuación Lingüística: perspectivas semánticas y pragmáticas (CIAL) en Valencia el 15-18 de julio en 2016.
} 
the context of impositive declarations, explanations, questions, indirect requests, suggestions, and offers.

KEYWORDS: conditional structures, politeness, mitigation

\section{$1 \quad$ INTRODUCCIÓN}

Numerosos estudios han considerado la fuerza atenuadora de las oraciones condicionales en inglés, español y otros idiomas (Levinson, 1983; Ford y Thompson, 1986; Dancygier, 1990; Sweetser, 1990, Montolío, 1992, 1993; inter alia). Chodorowska-Pilch $(1999,2001)$ propone un análisis dentro del marco de la gramaticalización ${ }^{2}$ y la cortesía del marcador si quieres, cuya fuerza atenuadora se manifiesta en las ofertas y sugerencias corteses.

Este trabajo pretende hacer una contribución muy puntual, usando los datos recogidos en una agencia de viajes en Valencia en el verano del $2000^{3}$ durante dos meses, de la mitad de junio a la mitad de agosto. Proponemos estudiar solo las estructuras condicionales en la conversación, cuya finalidad es atenuar la fuerza del contenido proposicional de ciertos actos de habla. Se demostrará que el uso de las estructuras condicionales se da en determinados contextos. La motivación de su uso se debe a la percepción del hablante, quien sigue las normas de cortesía (Lakoff 1973; Leech 1983; Brown y Levinson 1987) con el fin de mitigar la imposición de sus enunciados.

Para alcanzar este fin, se analizarán conversaciones coloquiales grabadas en una agencia de viajes con atención al uso de las construcciones condicionales como atenuantes pragmáticos que invocan la cortesía. Al mismo tiempo, se revisarán las construcciones condicionales como mecanismos gramaticales que sirven para mantener la imagen positiva y/o la negativa del oyente.

Observemos las interacciones en (1) y (2):

1) $\mathrm{Am}^{4}:$... y os dan una excursión opcional a Versalles ... por si queréis ver $\vee$ ya como el día anterior habéis visto un poco $\mathrm{P}$... pues nada [ ] alojamiento dentro del parque y si lo desea ... opcionalmente una cena y espectáculo en laa... leyenda Búfalo Bill [ ]

\footnotetext{
${ }^{2}$ Véase la propuesta sobre la gramaticalización en Chodorowska-Pilch (1998).

${ }^{3}$ La recogida de datos y la investigación fueron posibles gracias a la beca del Ministerio de Asuntos Exteriores de España, la que se me concedió en el verano de 2000.

${ }^{4}$ Abreviaturas: Am- agente mujer, Ah- agente hombre, C- cliente, $\rightarrow$ - cuando el interlocutor interrumpe el turno del habla del locutor, = se solapan dos conversaciones al mismo tiempo, [ ] - irrelevante o fragmentos en los que se oye el teléfono o la conversación de otra mesa, \{\} - ininteligible o transcripción dudosa, C: ........... - conversación telefónica, MAYÚSCULAS- se levanta el tono de voz; mayor énfasis, , - pausa breve, ...- pausa mayor. Se usará letra en negrita para señalar lo analizado y por razones del anonimato no se usarán ni nombres de las personas ni de los lugares.
} 
2) Am: siíl te lo que \{\} en seguiditaaa si te parece te lo pongo aquí.

Podemos ver que la agente usa las condicionales de una manera no canónica y parcial $^{5}$ en estos ejemplos. En realidad, estas esctructuras no son necesarias desde la perspectiva de la gramática, sino más bien desde la perspectiva pragmática ${ }^{6}$. Es decir, la agente intenta atenuar las ofertas que hace a sus clientes, lo cual parece ser motivado por la voluntad de mantener la máxima de cortesía propuesta por Lakoff (1973) o Leech (1983), o por el deseo de salvar/proteger la imagen a la Brown y Levinson $(1978,1987)$ tanto del propio hablante como del oyente en estas situaciones.

Tomando en cuenta los estudios mencionados a continuación, se propone, de acuerdo con Montolío (1996), que las condicionales crean la posibilidad de expresar significados pragmáticos en la interacción. En la transmisión de la cortesía hacia el oyente/el interlocutor, el uso de determinadas construcciones subyace los principios de cortesía, tal y como lo proponían Lakoff (1973), Leech (1983) o Brown y Levinson (1978, 1987). La atenuación a través de las construcciones condicionales se produce gracias a su valor hipotético o de condicionalidad. Ellas expresan opciones en vez de hacer afirmaciones en los enunciados. ${ }^{7}$

\section{MARCO TEÓRICO}

En cuanto al concepto de atenuación o mitigación, Fraser (1980) propone que: "la mitigación conversacional es la modificación de un acto de habla: la reducción de ciertos efectos desagradables que tiene un acto de habla en el oyente". Además según el mismo autor, «la mitigación conversacional conlleva cortesía.» Briz (1998: 143), por su parte, indica que «la función más frecuente de la atenuación es la cortesía.»

Si se trata de las máximas o principios que subyacen la aplicación de la cortesía verbal, hay que mencionar a Lakoff (1973) con sus máximas: "No te impongas a tu interlocutor», "Da opciones» y «Refuerza los lazos de camaradería».Brown y Levinson $(1978,1987)$ introducen las nociones de imagen negativa; el deseo de mantener la autonomía de las acciones de uno e imagen positiva; ser aceptado por el interlocutor. Basándose en los datos en español, Hernández Flores (1999: 42) observa que «la razón de ser en las conversaciones coloquiales en castellano es la necesidad de mantener el balance entre las imágenes de los interlocutores8, es decir entre los respectivos deseos de autonomía y afiliación.»

Con respecto al valor lingüístico de las condicionales, Montolío (1996:340) señala que: "la expresión de opciones es una de las funciones discursivas más frecuentes de las condicionales: el ser portadoras de tal opcionalidad las convierte en estructuras muy adecuadas para formular ofertas y para mitigar en un sentido amplio". En un trabajo posterior,

\footnotetext{
${ }^{5}$ Montolío (1999) llama "periféricos" a algunos usos de las estructuras condicionales.

${ }^{6}$ Montolío (1999: 3685) hace la misma observación acerca de los usos corteses en general de las condicionales.

${ }^{7}$ Albelda Marco (2005), a parte de la cortesía, destaca otras funciones en conversaciones coloquiales.

${ }^{8}$ Chodorowska-Pilch (2008) propone que el uso de verás invoca tanto la imagen positiva como la negativa en la conversación.
} 
Montolío (1999) compara el uso de las condicionales indirectas con si us plau en catalán y s'il vous plait en francés, que son marcadores de cortesía por excelencia.

Expandiendo diferentes enfoques y planteamientos teóricos, Chodorowska-Pilch (1999, 2001) demuestra que la estructura condicional parcial, si quieres, es un marcador gramaticalizado que sirve para codificar la cortesía en ofertas y sugerencias. En el presente estudio, se estudiarán otros enunciados basados en la estructura con si. Se demostrará el valor atenuador o intensificador de estas cuasi-condicionales en determinados casos.

\section{DATOS: AGENCIA DE VIAJES}

Las interacciones grabadas en una agencia de viajes en Valencia ${ }^{9}$ dan evidencia del uso de varios tipos de expresiones basadas en la construcción con si, que se prestan como atenuantes en diferentes actos de habla.

\subsection{A ver sip}

El uso más frecuente lo forman las llamadas estructuras dialógicas con la referencia al oyente / interlocutor (A ver si + Verbo) que han sido encontradas en la mitad de los ejemplos analizados. Esta construcción está al servicio de la cortesía tanto positiva como negativa, lo que podemos ver en los ejemplos (3) y (4):

3) C1: Vale ... mírame a ver si puedes adelantarme el viajeee

4) C1: Míramelo a ver si hay el viaje...

Am: A ver... ¿salida día?

Los ejemplos citados son peticiones indirectas expresadas por la construcción a ver si .... precedidas por las formas imperativas del verbo mirar. Gracias a la estructura condicional, el cliente suaviza la fuerza de estas peticiones formulándolas como si fueran sugerencias, en vez de peticiones. En el ejemplo (5), tenemos una situación parecida:

5) C1: ese día no ... de todas formas quiero que me lo mires a ver si hay porque así, yo ya confirmo mi

calendario ... pero tú ya me entiendes ... yo lo tengo 'estranjiis' pero el mal es que me lo haya cambiado

El cliente pide a la agente que le busque algo, usando a ver si hay, como si fuera una pregunta indirecta, seguida por la justificación para atenuar la imagen negativa del interlocutor y la imagen positiva del locutor. En (6), que es una conversación entre una cliente y otra persona con la que habla por teléfono, la agente atenúa primero la sugerencia (a), la afirmación impositiva (b) y la pregunta indirecta (c) que hace:

\footnotetext{
6) Am: es pa la zonaaa zonaaa deee de lo que es laaa... es por... a ver... a ver espérate... a) a ver si podemos matizarlo todo un poquito más, venga y apuntar aquíí

C1: porque Luis, quiere quiere esta tarde ya a confirmarlo ¿sabes?
}

\footnotetext{
${ }^{9}$ Me gustaría agradecerle a una estudiante de posgrado de la Universidad de Valencia por transcribir estas grabaciones.
} 
Am: ah!... a ver...b) a ver si me he equivocado yo con

C3: [ ]

Am: no pero espera... tranqui... yo os lo voy pasando...

C3: [ ]

Am: a ver te dijo... c) a ver si te va alguno..., RP, V ese es el

que queríais vosotros

En (7), a ver introduce una condicional aparentemente canónica por lo cual no podemos distinguir el uso canónico del uso no canónico de esta estructura. Lo que sí se puede observar es que a ver mitiga la oferta de la agente.

7) Am: .... lo que si que puedo hacer de todas formas es mirártelo desde Barcelona

... y y ver si un BL a ver si hubiese algo más económico ...\{ $\}$ llegarías a $B$...

o ¿cómo lo véis?

Montolío (1999: 3678) indica que la estructura a ver si $+p$ sirve para expresar la esperanza, lo cual conlleva cortesía al proponer invitaciones, planes comunes o recomendaciones. Esta construcción con si tiene valor desiderativo y aparece en construcciones independientes; es decir, la prótasis no tiene relación con ninguna apódosis.

\subsection{Sip, $q$}

Según Montolío (1999: 3685), las llamadas condicionales periféricas tienen carácter metadiscursivo y no responden a la ley de contraposición. Los ejemplos de ellas presentamos en (8) y (9):

8) Am: Si quieres, te lo averiguo.

9) Am: Si quieres, lo podemos hacer.

(8) y (9) son ofertas corteses dirigidas a los clientes. En vez de oraciones declarativas o preguntas, la agente elige la estructura condicional para mitigar la imposición de la oferta y salvaguardar la imagen de ambos participantes de la conversación.

\subsection{3. Sip}

Otro tipo forman las condicionales suspendidas, que solo contienen prótasis periféricas en el imperfecto de subjuntivo o presente indicativo, como en los enunciados en (10):

\footnotetext{
10) C1: Me decía Amparo, madre mía este año me va a entrar depresión porque ella es el primer año que ha ido, jay! Isabel yo es que cada vez que me acuerdo del T, yyy y si fuéramos a C el sitio lo buscan ellos

Am: ayer ya me estuvo diciendooo que si miramos otrooo le gusta aquí, porque Amparo sólo busca los de página grandee digo claro Amparo así como una tonta... jaja

C1: ella busca los de páginaaa ... donde esta ....

Am: a ver si es Costa Blancaaa la tuya va a estar aquí abajo hacia M... todo allí está abajo
}

En (10), tanto la cliente («si fuéramos») como la agente («si miramos») usa la prótasis de la estructura condicional, pero su uso no sirve para expresar un valor hipotético sino más bien 
expresan cierta posibilidad u opción, lo cual atenúa la fuerza de las sugerencias o afirmaciones impositivas. En el ejemplo (11), observamos un uso muy parecido al del (10):

11) Am: La de veces que llevo mirando esto, pero bueno, si lo tuviéramos, le preguntamos por la zona a Ana, que nos diga lo que tiene y luego ya decidimos

En (11), el uso de la prótasis en el imperfecto de subjuntivo mitiga la fuerza del enunciado de tal manera que la oferta suena como si fuera una sugerencia por parte de la agente, lo cual invoca la imagen positiva de la agente y la imagen negativa del cliente.

\subsection{Si quieres como marcador discursivo}

Las conversaciones de este corpus también corroboran el uso de «si quieres» como marcador de cortesía en la posición medial de las ofertas, como en (12) y (13):

12) Am: Coja, si quiere, una silla de mi compañera.

13) 'C1: noo primero voy a preguntar a ver que me dejan tomarme Am: ¿Vale?

$\rightarrow$ C1: y cambiaaar si quieres eso te llamo tee lo confirmo y ya está

Am: y ésta a ver qué pasa

C1: porque [ de vacaciones ... ¿y no saldrá ninguna oferta del puente?

Am: no ... agostooo y puenteee y quieres oferta ... ella ella per si acàs

C1: jyo lo preguntooo! Si fuera otro sitio no

En el ejemplo (13), además del marcador si quieres basado en la estructura condicional, también hay un uso de la condicional suspendida con la prótasis Si p, como en (11). Aquí, la cliente intenta mitigar una pregunta directa después de haber sido regañado por la agente. La cliente justifica el haberse atrevido a preguntar por una oferta en una temporada en que no suele haber ofertas. La agente usa la construcción per si acàs en valenciano de una manera irónica, sin que le importe salvaguardar la imagen negativa de su cliente. Podemos observar que es un uso descortés de esta construcción, lo cual queda fuera de nuestro enfoque en este estudio. La cliente intenta atenuar su imposición al usar la prótasis si fuera, que en este caso le sirve como una justificación de una afirmación impositiva. Este uso de la condicional parcial parece invocar principios de la cortesía y sobre todo la protección de la imagen negativa del cliente y la de la agente.

\subsection{Por sip}

En contraste al uso de per si acàs en valenciano en el ejemplo (13), la estructura por si p puede servir para salvaguardar la imagen negativa del cliente y reforzar la imagen positiva de la agente, como en el ejemplo (1), aquí repetido en (14):

14) Am: ... y os dan una excursión opcional a Versalles ... por si queréis ver $V$ ya como el día anterior habéis visto un poco P ... pues nada [ ] alojamiento dentro del parque y si lo desea ... opcionalmente una cena y espectáculo en laa... leyenda Búfalo Bill [ ] 
La construcción por si p sirve para mitigar una oferta, salvaguardando la imagen negativa del cliente. Cabe notar que, a diferencia de per si acàs en (13), el uso cortés de la estructura por si $\mathrm{p}$ involucra toda la oración que contiene el verbo querer que conlleva el valor de una posibilidad. También, el tratamiento del cliente en tercera persona apoya un trato más respetuoso. De esta manera, la agente se asegura de no imponer su oferta al cliente, tratando de mantener los principios de cortesía en la interacción con él, lo cual invoca las normas de cortesía de Lakoff (1973) o la teoría de Brown y Levinson (1978, 1987).

\subsection{Que sip}

Las conversaciones en la agencia de viajes también indican que la estructura condicional que si p (un sustantivo o una cláusula) puede expresar una actitud cortés por parte del hablante. Según Montolío (1999: 3683), la estructura "Que si + sustantivo / Que si + cláusula» es una estructura citativa con valor enfático. Su significado depende del contexto, ya que puede conllevar tanto el valor de cortesía como de descortesía. Veamos el ejemplo (15):

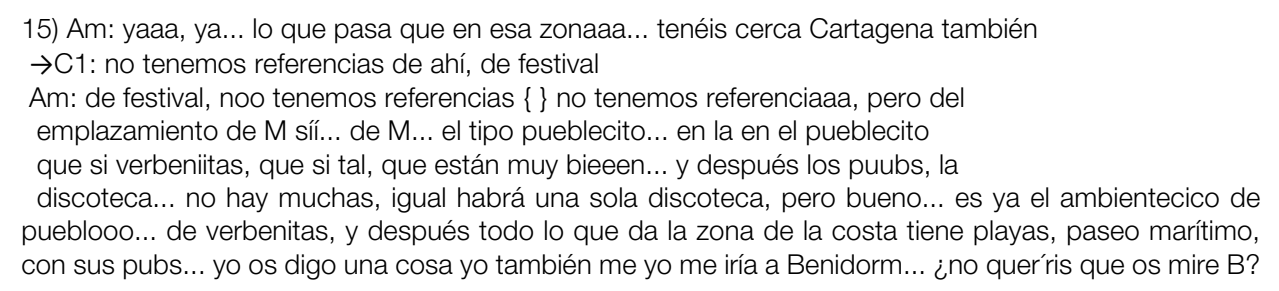

Lo que corrobora la actitud cortés de la estructura condicional que si p en este ejemplo es el uso de varios diminutivos (pueblecito, ambientecico) que invocan la cortesía positiva por parte de la agente, quien disfraza su oferta por el uso de estos recursos lingüísticos e invoca los principios de cortesía. Por lo tanto, la estructura que si p también sirve para mitigar una oferta y salvaguardar la imagen positiva de la agente y la negativa del cliente.

\subsection{Sip que $v /$ sip $+v$}

Las ofertas corteses son codificadas también por la estructura condicional parcial seguidas por una cláusula subordinada o una oración declarativa en vez de una pregunta directa hacia el oyente, como en el ejemplo (2), repetido aquí en (16), y en (17):

\footnotetext{
16) Am: siili te lo que \{\} en seguiditaaa si te parece te lo pongo aquí.

17) Am: jaaa, jah!... A, A... ¿sabes qué pasa? queee la zona $A$ de $C B$ cuando tienes

menos de siete noches te sale más caro... si quieres que mire ahora $\mathrm{S}$, ques

$C D$... en la CD pasa lo mismo... voy a mirarte mientras esto veintiuno veintidooos, sólo en $B$
}

En (16), la agente pudo haber dicho "¿Te lo pongo aquí?» o en (17), «iTe miro S?». Sin embargo, la agente usa la estructura condicional para indicar opcionalidad para el cliente y evitar imposición. Es importante destacar el uso de los verbos parecer y querer en esta estructura cuasi-condicional, los que corroboran la estrategia mitigadora de las ofertas o sugerencias. El deseo de evitar la imposición en la interacción, expresado por la estructura 
condicional y otros mecanismos lingüísticos (e.g. diminutivos), una vez más corrobora una estrategia deliberada por parte de los agentes como de los clientes en los datos en las agencias de viaje. Los que usan la estructura condicional de una manera no canónica y parcial parecen ser motivados por la observación de los principios de cortesía.

El si de la estructura condicional atenúa la fuerza en los enunciados que podrían amenazar la imagen negativa del oyente y refuerzan o protegen la imagen positiva de los hablantes, y por ello sirven como una estrategia para señalar la actitud cortés de los hablantes.

\section{RESUMEN Y RESULTADOS}

El análisis de los ejemplos relevantes de las agencias de viaje corrobora los resultados de los estudios anteriores sobre el uso de las condicionales y a la vez recoge una gama de usos de la construcción condicional al servicio de la expresión de la cortesía en español peninsular. Podemos proponer que las construcciones condicionales pueden servir para marcar la cortesía en los siguientes casos:

1) Estructuras dialógicas (A ver si p) en las peticiones, sugerencias y preguntas indirectas.

2) Condicionales periféricas (si p, q) en las ofertas.

3) Condicionales suspendidas (si p) como prótasis corteses con aspecto formulario10 o prótasis seguidas por un verbo en el imperfecto de subjuntivo o presente indicativo en las sugerencias o afirmaciones impositivas.

4) Prótasis gramaticalizada (si quieres) como marcador de cortesía11 en la posición medial y final de los enunciados en las ofertas y sugerencias.

5) Por si p para expresar una opción en las ofertas, sugerencias o afirmaciones impositivas.

6) Condicional parcial seguida por una cláusula subordinada o una oración declarativa en vez de una pregunta directa (Si p que v/si $p+v$ ) en las ofertas y preguntas indirectas.

7) Estructura condicional citativa (Que si + sustantivo / Que si + p)12 en las ofertas. Esta estructura depende del contexto; puede tener tanto el valor de cortesía como de descortesía.

Los ejemplos analizados aquí demuestran que el uso basado en las estructuras condicionales sirve para mitigar los enunciados que pueden resultar impositivos en las conversaciones. Al atenuarlos, los hablantes siguen los principios de la cortesía verbal propuestos por Lakoff (1973) o Brown y Levinson $(1978,1987)$.

\footnotetext{
${ }^{10}$ Montolío (1999: 3684) las compara con si us plau en catalán o s'il vous plaît en francés.

${ }^{11}$ Véanse más ejemplos en Chodorowska-Pilch (1999).

${ }^{12}$ Véase también Montolío (1999: 3683).
} 


\section{CONCLUSIONES PRELIMINARES}

Basándonos en los datos recogidos en una agencia de viajes durante dos meses, podemos constatar que las construcciones condicionales, que se usan para codificar cortesía, se pueden eliminar de los enunciados o reemplazarlas por otras estructuras sin afectar el significado proposicional del mismo. Sin embargo, estas condicionales son relevantes desde una perspectiva pragmática ya que permiten codificar una actitud más cortés de los hablantes, gracias a la atenuación de los enunciados impositivos. Las construcciones condicionales pueden invocar tanto la imagen positiva para mantener una buena relación con el oyente como la imagen negativa de uno o de los dos participantes de la conversación.

Las estructuras condicionales, que no hacen referencia gramatical al interlocutor, dependen del entorno lingüístico del enunciado, como es el caso de la estructura que si. Por su estructura sintáctica y su valor hipotético o de condicionalidad (el aspecto semántico), las condicionales sirven para codificar el significado pragmático (la cortesía). Es decir, el uso de ellas implica una estrategia compleja para atenuar los enunciados con el fin de mantener la imagen positiva y/o negativa.

En las conversaciones analizadas, las condicionales o cuasi-condicionales han sido usadas para mitigar/atenuar las ofertas, sugerencias, peticiones indirectas, afirmaciones impositivas y preguntas indirectas. Es posible que, en un entorno diferente, el uso de las condicionales no canónicas sea más extenso o más reducido, pero este hecho no niega que los hablantes pueden usar las condicionales como una estrategia para atenuar sus enunciados con el motivo de mantener los principios de cortesía.

\section{REFERENCIAS BIBLIOGRÁFICAS}

AlbeldA MARCO, Marta (2005): «Discordancia entre atenuación/cortesía e intensificación/descortesía en conversaciones coloquiales", en Blas, J. L., Casanova, M., Velando, M. (eds.): Discurso y Sociedad. Contribuciones al studio de la lengua en contexto social, Servicio de publicaciones de la Universidad de Castellón, 581-590.

BRIz GómEZ, Antonio (1998): El español coloquial en la conversación. Esbozo de pragmagramática, Barcelona, Ariel.

Brown, Penelope y Stephen LeVInson (1987 [1978]): Politeness: Some Universals in Language Usage, Cambridge: Cambridge University Press.

CHODOROWSKA-PILCH, Marianna (1998): Encoding of politeness in Spanish and Polish: A crosslinguistic study. University of Southern California dissertation. Ann Arbor (Michigan): University Microfilms International.
CHOdOROWSKA-PILCH, Marianna (1999): "Si quieres as a grammaticalized politeness marker in Peninsular Spanish» en ShinJa J. Hwang y Arle R. Lommel: LACUS Forum 25. LACUS, Fullerton, CA, 151-160.

CHODOROWSKA-PILCH, Marianna (2001): "Si quieres como marcador gramaticalizado de cortesía» en H. Ferrer y S. Pons (eds.): La pragmática de los conectores y las partículas modales. Universitat de València: Quaderns de Filología. Estudis Lingüístics, Vol. VI, 81-92.

CHODOROWSKA-PILCH, Marianna (2008): «Verás in Peninsular Spanish as a grammaticalized discourse marker invoking positive and negative politeness». Journal of Pragmatics 40/8: $1357-$ 1372.

DANCYGIER, Barbara (1990): "Conditionals: Sequence of events and sequence of clauses» en 
Jacek Fisiak (ed.): Further insights into contrastive analysis. Amsterdam, John Benjamins, 357-373.

ForD, Cecilia E. y Sandra A. THOMPSON (1986): "Conditionals in discourse: A text-based study from English" en E. C. Traugott (ed.): On conditionals. Cambridge: Cambridge University Press, 353-372.

FRASER, Bruce (1980): «Conversational mitigation». Journal of Pragmatics, 4, 341-350.

HERNÁNDEZ FLORES, Nieves (1999): «Politeness ideology in Spanish colloquial conversations: The case of advice.» Pragmatics 9 (1), 37-49.

LAKOFF, Robin (1973): "The Logic of Politeness; or, Minding Your P's and Q's.» Papers from the Ninth Regional Meeting of the Chicago Linguistic Society, 292-305.

LEECH, Geoffrey (1983): Principles of Pragmatics. London, Longman.

LeVINSON, Stephen C. (1983): Pragmatics. Cambridge, Cambridge University Press.

MONTOLío, Estrella (1992): «La teoría de la Relevancia y el procesamiento de las estructuras condicionales». En C. Martín Vide: Actas del VII Congreso de Lenguajes Naturales y Lenguajes Formales. Barcelona, Publicaciones y Promociones Universitarias, 453-59.

Montolío, Estrella (1993): «Si me lo permiten... Gramática y pragmática; sobre algunas estructuras condicionales regulativas en español» en H. Haverkate, K. Hengeveld y G. Mulder: Diálogos Hispánicos, vol. 12: Aproximaciones pragmalingüísticas al español. Amsterdam, Rodopi, 119-147.

MONTOLío, Estrella (1996): «Gramática e interacción (Ensayo metodológico para el análisis del español conversacional)». en A. Briz et al. (eds.): Pragmática y gramática del español hablado. Universidad de Valencia: Libros Pórtico, 329-341.

MONTOLío, Estrella (1999): "Las construcciones condicionales.» en I. Bosque y V. Demonte (eds.): Gramática descriptiva de la lengua española. 3. Entre la oración y el discurso. Morfología. Madrid: Espasa Calpe, S.A.

MolineR, María (1992): Diccionario de uso del español. Gredos, Madrid.

SWEETSER, Eve (1990): From etymology to pragmatics. Cambridge: Cambridge University Press.

Vigara TAuste, Ana María (1992): Morfosintaxis del español coloquial. Madrid: Gredos. 